\title{
Energy of weak gravitational waves in spacetimes with a positive cosmological constant
}

\author{
P.T. Chruściel, ${ }^{1, *}$ Sk Jahanur Hoque, ${ }^{2, \dagger}$ and Tomasz Smołka ${ }^{3, *}$ \\ ${ }^{1}$ University of Vienna, Faculty of Physics, Boltzmanngasse 5, A1090 Wien, Austria \\ ${ }^{2}$ Institute of Theoretical Physics, Faculty of Mathematics and Physics, \\ Charles University, V Holešovičkách 2, 18000 Prague 8, Czech Republic \\ ${ }^{3}$ Department of Mathematical Methods in Physics, Faculty of Physics, \\ University of Warsaw, Pasteura 5, PL 02-903 Warszawa, Poland
}

(Dated: February 2, 2021)

\begin{abstract}
We derive a formula for the total canonical energy, and its flux, of weak gravitational waves on a de Sitter background.
\end{abstract}

In view of the recent gravitational waves detections, and of the measurement of a positive cosmological constant, there arises an urgent need of a thorough understanding of gravitational waves in spacetimes with a positive cosmological constant. An important starting point for such studies is the understanding of weak gravitational waves in this context, as these are the ones that are seen by the detectors. One of the key issues is to determine how much energy is carried away by these gravitational waves.

An attempt to provide an answer to this question using the full nonlinear Einstein equations has been made in [5]. There a formula for the energy à la Trautman-Bondi has been derived, which turned out to be ambiguous because of a renormalised volume involved. The question then arises, whether the ambiguities can be resolved in some cases, e.g. for weak field configurations.

For this, let us consider an astrophysical system emitting gravitational waves, e.g. a collection of clusters of galaxies in a binary or in a localised many-body system; here "localised" should be understood in terms of cosmological scales. At these scales the resulting gravitational waves can be well approximated by linearised fields on a cosmological background except on a cosmologically-negligible region in an immediate neighborhood of the emitting system. And without any doubt, for such systems the field in the radiation zone is well described by the linearised theory. As a first approximation to the problem at hand it appears reasonable to consider solutions of the linearised vacuum equations throughout. The aim of this note is to derive a formula for the energy emitted in such a setting in the simplest cosmological model with a positive cosmological constant, namely de Sitter spacetime.

More precisely, we will calculate the canonical energy of weak gravitational waves on light cones in a de Sitter universe, and their flux.

Now, the worldline of an isolated system as above is well described by a timelike geodesic in de Sitter spacetime. Such geodesics are orbits of a Killing vector field, say $X$, which is timelike along this geodesic and tangent to it. A first guess would be that at any moment of time along the geodesic, the total energy contained in the gravitational wave emitted equals the integral, over the light-cone with vertex on the geodesic, of the canonical energy-momentum tensor of the gravitational

\footnotetext{
* piotr.chrusciel@univie.ac.at

$\dagger$ jahanur.hoque@utf.mff.cuni.cz

† tomasz.smolka@fuw.edu.pl
}

waves contracted with the vector field describing the motion of the light-cone, which in this case is the Killing vector field $X$. However, linearised gravitational waves are defined up to gauge transformations, and the question of a physically meaningful choice of the gauge arises. Whatever the gauge, the canonical energy-momentum tensor contracted with a Killing vector field of the de Sitter background provides a current with vanishing divergence, with the integral of this current over a three-dimensional hypersurface providing an associated charge. This is an integral over a region of infinite extent, and will not even be finite when a random gauge is chosen. So a minimal requirement is to use gauges which allow one to obtain, or at least identify (as will be done below), finite integrals.

In the case of vanishing cosmological constant, a good gauge for the purpose has been found by Bondi and collaborators $[4,9]$. In the nonlinear theory it leads to the Bondi energy, as well as the associated Trautman-Bondi mass loss formula [12]. It provides an extremely convenient coordinate system to analyse the solutions of the characteristic constraint equations. For linearised gravitational waves and $\Lambda=0$ the canonical energy, when calculated in the Bondi gauge, reproduces the Bondi energy and the Trautman-Bondi mass loss formula. It is therefore reasonable to expect that the use of the Bondi gauge will also provide a meaningful definition of total energy of weak gravitational waves in the presence of a positive cosmological constant. This is the approach taken here. After isolating terms which would lead to infinite energy and which have a dynamics of their own, one is thus led to our formula (23) below for the total energy of weak gravitational waves contained in a light cone, together with the formula (24) for the flux of energy when the cones are dragged along a timelike geodesic.

We note that in linearised gravity the energy, as defined by the procedure below, does not depend on the gauge inside the spacetime, but only on the asymptotic behaviour of the gauge functions. So the Bondi gauge is relevant for the analysis only insofar it allows one to provide explicit formulae for a few leading components of the asymptotic expansion of the metric, and give concise expressions for the integrals involved.

This work is related to that in [1,2], where a definition of energy is used which differs from the one used here by boundary terms. This difference is irrelevant when integrating over compact boundaryless surfaces. However, as made clear below, a proper inclusion of boundary terms is important to obtain the energy flux formulae that we are about to derive. We emphasise that keeping track of these terms is crucial already in the 
case $\Lambda=0$, whether in the linearised case addressed here or in the full nonlinear setting.

It should be kept in mind that an approximate solution describing a gravitational wave emitted by a gravitating system has been derived in [3]. Unfortunately, this solution is singular near the vertex of the light cone. This is not an issue for the analysis there, since the authors of [3] are concerned with the large-distance behaviour of the solution. However, such solutions are not suitable in our context: while the knowledge of the asymptotics of the field suffices to obtain the flux formula for energy, the solution needs to be put into the Bondi gauge to calculate our energy flux. This requires regularity everywhere, including the vertex. It would be of interest to extend the analysis in [3] to obtain globally regular solutions.

Recall that a metric in Bondi coordinates takes the form

$$
\begin{aligned}
g_{\alpha \beta} d x^{\alpha} d x^{\beta} & =-\frac{V}{r} e^{2 \beta} d u^{2}-2 e^{2 \beta} d u d r \\
& +r^{2} \gamma_{A B}\left(d x^{A}-U^{A} d u\right)\left(d x^{B}-U^{B} d u\right),
\end{aligned}
$$

where $\left(x^{A}\right) \equiv\left(x^{2}, x^{3}\right) \equiv(\theta, \varphi)$, together with the condition

$$
\operatorname{det} \gamma_{A B}=\sin ^{2} \theta \text {. }
$$

The de Sitter metric can be written in this form (cf., e.g., [6])

$$
\begin{aligned}
g & \equiv g_{\alpha \beta} d x^{\alpha} d x^{\beta} \\
& =\underbrace{-\left(1-\Lambda r^{2} / 3\right)}_{=: \epsilon N^{2}} d u^{2}-2 d u d r+r^{2} \underbrace{\left(d \theta^{2}+\sin ^{2} \theta d \phi^{2}\right)}_{=: \gamma},
\end{aligned}
$$

with $\epsilon \in\{ \pm 1\}$ determined by the sign of $g_{u u}$. We consider solutions, say $h_{\mu v}$, of the linearised vacuum Einstein equations which, in the coordinate system of (3), satisfy the gauge conditions resulting from (1)-(2):

$$
h_{r r}=0=h_{r A}, \quad \dot{\gamma}^{A B} h_{A B}=0 .
$$

The equations satisfied by $h_{\mu \nu}$ can be derived from the Lagrangian obtained by taking the quadratic part of $\frac{\sqrt{|\operatorname{det} g|}}{16 \pi}(R-$ $2 \Lambda$ ) and discarding a divergence,

$$
\mathscr{L}[h]=\frac{1}{32 \pi} \sqrt{|\operatorname{det} g|}\left(P^{\alpha \beta \gamma \delta \epsilon \sigma} \nabla_{\alpha} h_{\beta \gamma} \nabla_{\delta} h_{\epsilon \sigma}+Q(h)\right),
$$

where $Q$ is a quadratic polynomial in $h$,

$$
Q(h)=\frac{2 \Lambda}{(d-2)}\left[g^{\alpha \rho} g^{\beta \sigma} h_{\alpha \beta} h_{\rho \sigma}-\frac{1}{2}\left(g^{\alpha \beta} h_{\alpha \beta}\right)^{2}\right]
$$

with $d=4$ here, and

$$
\begin{gathered}
P^{\alpha \beta \gamma \delta \epsilon \sigma}=\frac{1}{2}\left(g^{\alpha \epsilon} g^{\delta \beta} g^{\gamma \sigma}+g^{\alpha \epsilon} g^{\sigma \beta} g^{\gamma \delta}-g^{\alpha \delta} g^{\beta \epsilon} g^{\sigma \gamma}-g^{\alpha \beta} g^{\gamma \delta} g^{\epsilon \sigma}\right. \\
\left.-g^{\beta \gamma} g^{\alpha \epsilon} g^{\sigma \delta}+g^{\beta \gamma} g^{\alpha \delta} g^{\epsilon \sigma}\right)
\end{gathered}
$$

(only a suitable symmetrisation of $P^{\alpha \beta \gamma \delta \epsilon \sigma}$ is relevant, as follows from the structure of (5)). Given a Lagrangian field theory of fields $\phi^{A}$ with Lagrangian $\mathscr{L}$, the Hamilton function associated with a vector field $X$ and a hypersurface $\mathscr{S}$ is defined as the integral

$$
\mathscr{H}[\mathscr{S}, X, \phi]:=\int_{\mathscr{S}}(\underbrace{\frac{\partial \mathscr{L}}{\partial \phi^{A}} \mathcal{L}_{X} \phi^{A}-X^{\mu} \mathscr{L}}_{=: \mathscr{H}^{\mu}}) d S_{\mu},
$$

where $\phi^{A}{ }_{\mu}:=\partial_{\mu} \phi^{A}$. The functional $\mathscr{H}[\mathscr{S}, X, \phi]$ is usually a starting point for calculating the Hamiltonian generating the dynamics of the theory, and equals this Hamiltonian in many cases. We shall, however, not pursue this line of thought as it involves considerations which are irrelevant for our purposes here.

Let $\mathscr{C}_{u}$ denote a level set of the coordinate $u$ as in (3), this is a light cone emanating from $r=0$. The light cones $\mathscr{C}_{u}$ are thus obtained by moving the tip of an initial light cone $\mathscr{C}_{u_{0}}$ to the future with the vector field $\partial_{u}$. In spacetimes which are asymptotically flat in null directions, for which $\Lambda=0$, the numerical value of $\mathscr{H}\left[\mathscr{C}_{u}, \partial_{u}, \phi\right]$ would be identified with the total energy of the field contained in $\mathscr{C}_{u}$. This motivates the use of the term "energy" when $X=\partial_{u}$. While the vector field $\partial_{u}$ is timelike near the tips of the $\mathscr{C}_{u}$ 's, it is not at large distances. This last feature, unavoidable in our context, is somewhat unusual for energy for field theory on Minkowski spacetime, but we are not on Minkowski spacetime.

If we extend our model to one which allows matter fields concentrated near the tip of the light cone, then their contribution to the canonical energy will be the usual energy associated with a Killing vector of the background which is timelike where matter is located. This makes clear the sense in how our flux formula would include a contribution from the usual energy of matter fields.

If attempting to carry-out our construction on a general background we would take $X$ to be that Jacobi field (also known as "geodesic deviation" vector field) along the generators of the $\mathscr{C}_{u}$ 's which propagates these generators to the future without rotation at the tip; in de Sitter spacetime this Jacobi field turns out to be the restriction to $\mathscr{C}_{u}$ of the Killing vector $\partial_{u}$.

Let $\mathscr{C}_{u, R}$ denote the part of the light cone $\mathscr{C}_{u}$ which extends from the tip to the Bondi-coordinate-radius $r=R$. Given a smooth solution $h_{\mu \nu}$ of the linearised vacuum Einstein equations one can find a Bondi gauge such that for small $r$ we have ${ }^{1}$ on $\mathscr{C}_{u, R}$

$$
h_{A B}=O\left(r^{2}\right) .
$$

In the gauge (9) one finds the following formula for the canonical energy $E_{c}\left[h, \mathscr{C}_{u, R}\right]$ :

$$
\begin{aligned}
& E_{c}\left[h, \mathscr{C}_{u, R}\right]:=\mathscr{H}\left[\mathscr{C}_{u, R}, \partial_{u}, h\right] \\
& =\frac{1}{64 \pi} \int_{\mathscr{C}_{u, R}} g^{B E} g^{F C}\left(\partial_{u} h_{B C} \partial_{r} h_{E F}-h_{B C} \partial_{r} \partial_{u} h_{E F}\right) r^{2} d r d^{2} \mu_{\gamma} \\
& \quad-\frac{1}{32 \pi} \int_{S(R)} P^{r(\beta \gamma) \delta(\epsilon \sigma)} h_{\beta \gamma} \nabla_{\delta} h_{\epsilon \sigma} d^{2} \mu_{\gamma}
\end{aligned}
$$

where $S(R)$ denotes a sphere of radius $R$ and

$$
d^{2} \mu_{\gamma}=\sin (\theta) d \theta d \varphi .
$$

(In a Bondi gauge where (9) does not hold this formula cannot be used, because the fields transformed to Bondi coordinates are then too singular at the origin.)

\footnotetext{
${ }^{1}$ We note that (9) will typically not be preserved by evolution when asymptotic gauge conditions such as e.g. (12) are imposed, and that on nearby light cones we will then only have $h_{A B}=O(r)$ for small $r$ in general.
} 
Some readers might worry that nonlinear effects will destroy the smoothness of the light cones, making our construction inadequate for the purpose. This is not the case in spacetimes arising from sufficiently small, fully nonlinear, perturbations on a Cauchy surface $\mathscr{S}$, for light cones emanating from points lying to the future of $\mathscr{S}$, as follows from [7].

To continue one needs to understand the asymptotic behaviour of the fields for large $r$. In that region it is convenient to define the rescaled fields

$$
\check{h}_{\mu v}:=r^{-2} h_{\mu v},
$$

keeping in mind that the symmetric trace-free tensor field $h_{A B}$ on a light cone is freely prescribable. It follows e.g. from [7] (compare [5, Proposition 2.1]) that there exists a dynamically consistent class of fields $\breve{h}_{A B}$ which have an asymptotic expansion of the form, for large $r$,

$$
\begin{aligned}
\check{h}_{A B}\left(u, r, x^{C}\right)= & \frac{\stackrel{(-1)}{h}_{A B}\left(u, x^{C}\right)}{r}+\frac{\stackrel{(-2)}{h}_{A B}\left(u, x^{C}\right)}{r^{2}}+\frac{\stackrel{(-3)}{\breve{h}}_{A B}\left(u, x^{C}\right)}{r^{3}} \\
& +\ldots,
\end{aligned}
$$

where the coefficients of the expansion $\stackrel{(-1)}{h}_{A B}$, etc., do not depend upon $r$. Existence of a smooth conformal completion requires $\stackrel{(-2)}{h}_{A B} \equiv 0$ [5], and we emphasise that this is consistent with the evolution equations. The remaining $h_{\mu \nu}$ 's are determined by the linearised version of the characteristic constraint equations in Bondi coordinates [8], this proceeds as follows. First, these equations give, in vacuum, $\partial_{r} h_{r u}=0$, and our choice of asymptotic gauge leads to

$$
h_{r u} \equiv 0 \text {. }
$$

Next we have

$$
\partial_{r}\left(r^{4} \partial_{r} \breve{h}_{u A}\right)=\underbrace{r^{2} \stackrel{\circ}{D}_{E}\left(\stackrel{\curlywedge}{\gamma}^{E F} \partial_{r} \check{h}_{A F}\right)}_{=: \psi_{A}},
$$

where $\stackrel{\circ}{D}_{A}$ is the covariant derivative of the unit round-sphere metric $\dot{\gamma}_{A B}$. Integrating in $r$ twice and using regularity of the metric at the vertex one obtains

$$
\begin{aligned}
\breve{h}_{u A}\left(u, r, x^{A}\right)= & \mu_{A}\left(u, x^{B}\right) \\
& +\int_{1}^{r}\left[\frac{1}{\rho^{4}} \int_{0}^{\rho} \psi_{A}\left(u, s, x^{A}\right) d s\right] d \rho,
\end{aligned}
$$

for a $u$-dependent family of covector fields $\mu_{A}$ on $S^{2}$, to be determined shortly from the asymptotic conditions. Equation (14) leads to the asymptotic expansion

$$
\check{h}_{u A}=\stackrel{(0)}{h}_{u A}+\frac{1}{2} \stackrel{ }{D}^{B} \stackrel{(-1)}{h}_{A B} r^{-2}+\stackrel{(-3)}{h}_{u A} r^{-3}+\ldots,
$$

with

$$
\stackrel{(-3)}{h}_{u A}=-\frac{1}{3} \lim _{r \rightarrow \infty}\left(\int_{0}^{r} \psi_{A}(s) d s+\stackrel{\stackrel{D}{D}}{B}^{(-1)} \stackrel{h}{h}_{A B} r\right),
$$

and we note that the limit is finite.

It follows from our boundary conditions and the evolution equations that the field $\stackrel{(0)}{h}$ uA is determined, up to a $u$-dependent family of conformal Killing vectors on the round sphere, from the free characteristic data $h_{A B}$ by the equation

$$
\stackrel{\circ}{D}_{A} \stackrel{(0)}{h}_{u B}+\stackrel{\circ}{D}_{B} \stackrel{(0)}{h}_{u A}-\stackrel{\circ}{D}^{C} \stackrel{()}{h}_{u C} \stackrel{\circ}{\gamma}_{A B}=-\frac{\Lambda}{3} \stackrel{(-1)}{h}_{A B}
$$

(This equation allows a non-zero field $\stackrel{(0)}{h}_{u A}$ even if $\Lambda=0$. In the asymptotically Minkowskian case it is consistent to assume $\stackrel{(0)}{h}_{u A} \equiv 0$, but it is not in general when $\Lambda>0$.) The field $\stackrel{(0)}{h}_{u A}$ determines the field $\mu_{A}$ appearing in (14). One finds the following form of the boundary term in (10):

$$
\begin{aligned}
& -\frac{\Lambda R}{192 \pi} \int_{S^{2}} \dot{\gamma}^{A B} \stackrel{\circ}{\gamma}^{C D} \stackrel{(-1)}{h}_{A C} \stackrel{(-1)}{h}_{B D} d^{2} \mu_{\gamma} \\
& -\frac{1}{64 \pi} \int_{S^{2}} \dot{\gamma}^{A B}\left(\dot{\circ}^{C D} \stackrel{(-1)}{h}_{A C} \partial_{u} \stackrel{(-1)}{h}_{B D}-6 \stackrel{(0)}{h}_{u A} \stackrel{(-3)}{h}_{u B}\right) d^{2} \mu_{\gamma} \\
& +o(1),
\end{aligned}
$$

where $o(1)$ denotes terms which tend to zero as $R$ tends to infinity, and where $S^{2} \equiv S(1)$ is the unit sphere.

The expression in (18) tends to minus infinity as $R \rightarrow \infty$ if $\stackrel{(-1)}{h}_{A C}$ is not identically zero, and begs the questions whether

1. the divergence of the boundary integral is compensated by that of the volume integral and, if not,

2. whether the boundary integral is needed at all in the definition of energy and, if so,

3. can one obtain consistent solutions by restricting oneself to a set of fields with $\stackrel{(-1)}{h}_{A C} \equiv 0$.

The answer to the second question is yes: if $\Lambda=0$, one will not obtain the Trautman-Bondi mass loss formula without this term.

To answer the remaining questions one needs to make use of the linearisation of the evolution equation for $g_{A B}$ [8, Equation (32)]: Denoting by

\section{$T S[\cdot]$}

the traceless symmetric part of a tensor, we have in vacuum

$$
r \partial_{r}\left(r \partial_{u} \breve{h}_{A B}\right)+\frac{\epsilon}{2} \partial_{r}\left(N^{2} r^{2} \partial_{r} \breve{h}_{A B}\right)-T S\left(\stackrel{\circ}{D}_{A}\left(\partial_{r}\left(r^{2} \breve{h}_{u B}\right)\right)\right)=0 .
$$

Integrating, we find

$$
\begin{aligned}
& \partial_{u} \breve{h}_{A B}(r, \cdot) \\
& =-\frac{1}{r} \int_{0}^{r} \frac{1}{s}\left(\frac{\epsilon}{2} \partial_{r}\left(N^{2} r^{2} \partial_{r} \breve{h}_{A B}\right)-T S\left(\stackrel{\circ}{D}_{A}\left(\partial_{r}\left(r^{2} \breve{h}_{u B}\right)\right)\right)\right)(s, \cdot) d s \\
& =\frac{\partial_{u} \stackrel{(-1)}{h}_{A B}(\cdot)}{r}+\frac{\partial_{u} \stackrel{(-3)}{h}_{A B}(\cdot)}{r^{3}}+o\left(r^{-3}\right),
\end{aligned}
$$

where

$$
\begin{aligned}
\partial_{u} \stackrel{(-1)}{h}_{A B}(\cdot)= & -\int_{0}^{\infty} \frac{1}{s}\left(\frac{\epsilon}{2} \partial_{r}\left[r^{2} N^{2}\left(\partial_{r} \check{h}_{A B}\right)\right]\right. \\
& \left.-T S\left[\stackrel{\circ}{D}_{A}\left(\partial_{r}\left(r^{2} \check{h}_{u B}\right)\right)\right]\right)(s, \cdot) d s .
\end{aligned}
$$


Convergence of this integral follows from the identity (17).

Equation (21) shows that there is no reason for $\partial_{u} \stackrel{(-1)}{h}_{A B}$ to vanish in general. Hence the field $\stackrel{(-1)}{\breve{h}}_{A B}$ will be nonzero at later times even if it was on some initial light cone unless the initial data are very special. Therefore the answer to question 3 . is clearly negative.

We note that one of the issues that arises in the asymptotically flat case is the contamination of the outgoing radiation field by incoming radiation. In our construction, both when $\Lambda=0$ or $\Lambda \neq 0$, the "no incoming radiation condition" can be implemented by, e.g., requiring the free data on some initial light cone to be compactly supported. Formula (21) shows that even such a strong assumption will not lead to a faster fall-off of the fields at later times in general.

Returning to our main argument, using (20) one finds a finite volume contribution to the canonical energy. So the volume integral cannot be used to compensate the divergence of the boundary integral. This answers the first question in the negative, and is rather worrisome.

A way out is provided by the flux formula satisfied by the energy. Indeed, if $X$ is a Killing vector of the background metric $g$ and if the field $h_{\mu v}$ satisfies the linearised Einstein equations, then the divergence of the field $\mathscr{H}^{\mu}$ vanishes. This leads to the following flux equation

$$
\begin{aligned}
& \frac{d E_{c}\left[h, \mathscr{C}_{u, R}\right]}{d u}= \\
& -\frac{\Lambda R}{96 \pi} \int_{S^{2}} \dot{\gamma}^{A B} \dot{\gamma}^{C D} \stackrel{(-1)}{h}_{A C} \partial_{u} \stackrel{(-1)}{h}_{B D} d^{2} \mu_{\gamma} \\
& -\frac{1}{32 \pi} \int_{S^{2}} \dot{\gamma}^{A B}\left(\stackrel{\circ}{\gamma}^{C D} \partial_{u} \stackrel{(-1)}{h}_{A C} \partial_{u} \stackrel{(-1)}{h}_{B D}-6 \stackrel{(-3)}{h_{h}} \partial_{u A} \partial_{u} \stackrel{(0)}{h}_{u B}\right) d^{2} \mu_{\gamma} \\
& +o(1) \text {. }
\end{aligned}
$$

This equation shows that the divergent term in $E_{c}$ has a dynamics of its own, evolving separately from the remaining part of the canonical energy. It is therefore natural to introduce a renormalised canonical energy, say $\hat{E}_{c}\left[h, \mathscr{C}_{u, R}\right]$, by removing the divergent term in (18). After having done this, we can pass to the limit $R \rightarrow \infty$ to obtain:

$$
\begin{aligned}
& \hat{E}_{c}\left[h, \mathscr{C}_{u}\right]:= \\
& \frac{1}{64 \pi} \int_{\mathscr{C}_{u}} g^{B E} g^{F C}\left(\partial_{u} h_{B C} \partial_{r} h_{E F}-h_{B C} \partial_{r} \partial_{u} h_{E F}\right) r^{2} d r d^{2} \mu_{\gamma}
\end{aligned}
$$

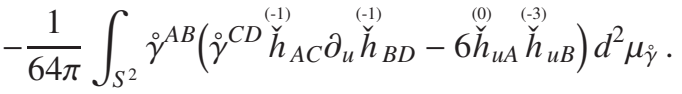

This is our first main result here, and is our proposal how to calculate the total energy contained in a light cone of a weak gravitational wave on a de Sitter background.

The flux equation for the renormalised energy $\hat{E}_{c}$ coincides with the one obtained by dropping the term linear in $R$ in (22) and passing again to the limit $R \rightarrow \infty$ :

$$
\begin{aligned}
& \frac{d \hat{E}_{c}\left[h, \mathscr{C}_{u, R}\right]}{d u}= \\
& -\frac{1}{32 \pi} \int_{S^{2}} \dot{\gamma}^{A B}\left(\stackrel{\circ}{\gamma}^{C D} \partial_{u} \stackrel{(-1)}{h}_{A C} \partial_{u} \stackrel{(-1)}{h}_{B D}-6 \check{h}_{u A} \partial_{u} \stackrel{(0)}{h}_{u B}\right) d^{2} \mu_{\gamma} .
\end{aligned}
$$

This is our key new formula. When $\Lambda=0$ we recover the weak-field version of the usual Trautman-Bondi mass loss formula since, as already pointed out, $\stackrel{(0)}{h}_{u A} \equiv 0$ in the asymptotically Minkowskian case. Hence the last term in (24) shows how the cosmological constant affects the flux of energy emitted by a gravitating astrophysical system.

Our mass loss formula has a $\Lambda$-dependent correction which can be both positive or negative, which is expected. Indeed, the de Sitter spacetime contains Cauchy hypersurfaces which are three-dimensional spheres, which implies that the total energy of gravitational waves in the full non-linear theory vanishes. Equivalently, in spatially closed universes the kinetic energy of the waves is exactly compensated by the negative potential energy arising from the self-interaction of the gravitational field. This implies in turn that any flux formula for energy must contain terms with indeterminate sign.

It would be of interest to compare (24) with the linearisedgravity version of the mass-evolution formula in [10]. We plan to leave this for future work. See also [11] for further references.

In Appendix A we provide an analysis of asymptotic symmetries. Our energy is invariant under those asymptotic symmetries which preserve (9). There remains the important question of the invariance of our energy under the remaining asymptotic symmetries. There is a difficulty here related to the fact that the gauge condition (9), which can be imposed on any given light cone and in which the formulae simplify, does not necessarily hold on nearby light cones. We plan to return to this question in the future.

Concluding, it should be admitted that we are still in a preliminary stage of understanding of the proper notion of energy in spacetimes with a positive cosmological constant. Our formula raises further questions concerning its uniqueness and its physical significance. This deserves further investigations. However, the analysis here gives a clear cut answer in a precise toy-model setting.

Acknowledgements: We heartfully thank Maciej Maliborski for checking the linearised Einstein equations. Useful discussions with P. Aichelburg, T. Damour, G. Date, J. Jezierski, M. Kolanowski, P. Krtouš and J. Lewandowski are acknowledged. JH is grateful to the Erwin Schrödinger Institute, and University of Vienna for hospitality during part of work on this paper. His research was supported in part by the Czech Science Foundation Grant 19-01850S, and by the DST Max-Planck partner group project "Quantum Black Holes" between Chennai Mathematical Institute, Siruseri, Tamilnadu, India and Albert Einstein Institute, Potsdam, Germany. TS acknowledges the hospitality of the University of Vienna during part of work on this project and financial support from the COST Action CA16104 GWverse. His work was supported by the University of Warsaw Integrated Development Programme (ZIP), co-financed by the European Social Fund within the framework of Operational Programme Knowledge Education Development 2016-2020, action 3.5. The research of PTC was supported by the Austrian Research Fund (FWF), Project P 29517-N27 and by the Polish National Center of Science (NCN) under grant 2016/21/B/ST1/00940. Last but not least, PTC is grateful to the Institut de Hautes Études Scientifiques, Bures-sur-Yvette, for hospitality during the final 
stage of work on this paper.

\section{Appendix A: Asymptotic symmetries}

An important question is the gauge freedom left. For this, one checks that vector fields $\stackrel{\zeta}{\zeta}$ generating the gauge transformations

$$
h_{\mu v} \mapsto h_{\mu v}+\nabla_{\mu} \dot{\zeta}_{v}+\nabla_{\nu} \dot{\zeta}_{\mu}
$$

and preserving the Bondi coordinate conditions as well as our asymptotic conditions take the form

$$
\begin{aligned}
\dot{\zeta}= & \left(\int \frac{\stackrel{\circ}{D}_{B} \xi^{B}\left(u, x^{A}\right)}{2} d u+\stackrel{\stackrel{\xi}{ }}{ }^{u}\left(x^{A}\right)\right) \partial_{u}+\left(\frac{\Delta_{j} \xi^{u}}{2}-\frac{r \stackrel{\circ}{B}_{B} \xi^{B}}{2}\right) \partial_{r} \\
& +\left(\xi^{B}\left(u, x^{A}\right)-\frac{1}{r} \stackrel{\circ}{D}^{B} \xi^{u}\left(u, x^{A}\right)\right) \partial_{B},
\end{aligned}
$$

with an arbitrary function $\stackrel{\circ}{\xi}^{u}\left(x^{A}\right)$ and where, at each $u$, $\xi^{B}\left(u, x^{A}\right) \partial_{B}$ is a conformal Killing vector field of $\dot{\gamma}$. These gauge transformations can be further constrained as follows: The Hodge-Kodaira decomposition of one-forms on $S^{2}$ shows that there exist functions $\hat{\chi}(u)$ and $\check{\chi}(u)$ on $S^{2}$ such that

$$
\stackrel{(0)}{h}_{u B}(u, \cdot)=\stackrel{\circ}{D}_{B} \hat{\chi}(u)+\varepsilon_{B}{ }^{C} \stackrel{\circ}{D}_{C} \check{\chi}(u),
$$

where $\varepsilon_{B}{ }^{C}$ is the two-dimensional Levi-Civita tensor on the round unit two-sphere. We can similarly write $\xi_{B}$ as

$$
\xi_{B}(u, \cdot)=\stackrel{\circ}{D}_{B} \iota(u)+\varepsilon_{B}^{C} \stackrel{\circ}{D}_{C} v(u),
$$

where the functions $\iota(u)$ and $v(u)$ are linear combinations of $\ell=1$ spherical harmonics. We have the following transformation law

$$
\stackrel{(0)}{h}_{u A}\left(u, x^{A}\right)+\stackrel{\circ}{\gamma}_{A B} \partial_{u} \stackrel{\circ}{\xi}^{B}\left(u, x^{A}\right)+\epsilon \frac{\Lambda}{3} \partial_{A} \xi^{u}\left(u, x^{A}\right),
$$

where $\epsilon$ is as in (3), which implies

$$
\begin{aligned}
\hat{\chi}(u) & \mapsto \hat{\chi}(u)+\partial_{u} \iota(u)+\frac{\Lambda}{3} \xi^{u}(u, \cdot), \\
\check{\chi}(u) & \mapsto \check{\chi}(u)+\partial_{u} v(u) .
\end{aligned}
$$

Let $P_{1}$ denote the $L^{2}\left(S^{2}\right)$-orthogonal projection on the space of $\ell=1$ spherical harmonics. We can arrange that $P_{1}(\check{\chi})$ vanishes by solving the linear ODE

$$
\partial_{u} v=-P_{1}(\check{\chi}),
$$

which leaves the freedom of choosing $v\left(u_{0}\right)$.

Next, using (A6), together with

$$
\partial_{u} \xi^{u}\left(u, x^{A}\right)=\frac{\stackrel{\circ}{D}_{B} \xi^{B}\left(u, x^{A}\right)}{2},
$$

we obtain

$$
\begin{aligned}
\partial_{u} \hat{\chi}(u) & \mapsto \partial_{u} \hat{\chi}(u)+\partial_{u}^{2} \iota(u)+\frac{\Lambda}{3} \partial_{u} \xi^{u}(u, \cdot) \\
& =\partial_{u} \hat{\chi}(u)+\partial_{u}^{2} \iota(u)-\frac{\Lambda}{3} \iota(u) .
\end{aligned}
$$

We can arrange that $\partial_{u}\left(P_{1}(\hat{\chi})\right)$ vanishes by solving the equation

$$
\partial_{u}^{2} \iota-\frac{\Lambda}{3} \iota=P_{1}\left(\partial_{u} \hat{\chi}\right)
$$

Equation (A6) shows that $P_{1} \hat{\chi}$ will vanish if

$$
\partial_{u} \iota\left(u_{0}\right)+P_{1}\left(\hat{\chi}\left(u_{0}\right)+\frac{\Lambda}{3} \xi^{u}\left(u_{0}, \cdot\right)\right)=0 .
$$

For example, Equation (A12) can be used to calculate $P_{1}\left(\xi^{u}\left(u_{0}, \cdot\right)\right)$ after prescribing $\partial_{u} \iota\left(u_{0}\right)$.

There remains thefore the freedom of choosing $\iota\left(u_{0}\right)$ and $\partial_{u} \iota\left(u_{0}\right)$, with the solutions of the homogeneous equation (A11) taking the form

$$
\iota(u, \cdot)=e^{\alpha u} \iota_{+}(\cdot)+e^{-\alpha u} \iota_{-}(\cdot),
$$

where $\iota_{ \pm}$are linear combinations for $\ell=1$ spherical harmonics.

Summarising, we can achieve a rigid transport of the Bondi coordinates from one sphere to the other by requiring that the potentials $\hat{\chi}$ and $\check{\chi}$ of (A4) satisfy

$$
P_{1}(\hat{\chi}) \equiv 0 \equiv P_{1}(\check{\chi})
$$

We will refer to (A14) as the rigid transport condition.

Once the rigid transport has been enforced, there remains the freedom of choosing $\left(\iota\left(u_{0}\right), \partial_{u} \iota\left(u_{0}\right), v\left(u_{0}\right)\right)$, which is related to the freedom of rotating and boosting the initial light cone $\mathscr{C}_{u_{0}}$, and of choosing $\xi^{u}\left(u_{0}, \cdot\right)$, which is the equivalent of the supertranslations that arise in the case $\Lambda=0$, subject to the constraint

$$
\partial_{u} \iota\left(u_{0}\right)+\frac{\Lambda}{3} P_{1}\left(\xi^{u}\left(u_{0}, \cdot\right)\right)=0 .
$$

After imposing (A14), the residual gauge transformations which also preserve the rigid transport condition (A14) take the form (A2) with an arbitrary function $\stackrel{\circ}{\xi}^{u}\left(x^{A}\right)$, and where $\stackrel{\circ}{\xi}^{B}\left(u, x^{A}\right) \partial_{B}$ is the angular part of a Killing vector field of de Sitter spacetime as in (A4), thus $v$ is a $u$-independent linear combination of $\ell=1$ spherical harmonics, the potential $\iota$ takes the form (A13), with $\partial_{u} \iota\left(u_{0}\right)$ satisfying (A15).

We will refer to these "infinitesimal coordinate transformations" as asymptotic symmetries.
[1] A. Ashtekar, B. Bonga, and A. Kesavan, Asymptotics with a positive cosmological constant: I. Basic framework, Class. Quan- tum Grav. 32 (2015), 025004, 41 pp., arXiv:1409.3816 [gr-qc]. MR 3291776 
[2] __ Asymptotics with a positive cosmological constant: II. Linear fields on de Sitter spacetime, Phys. Rev. D92 (2015), 044011, 14, arXiv:1506.06152 [gr-qc]. MR 3441014

[3] _ Asymptotics with a positive cosmological constant. III. The quadrupole formula, Phys. Rev. D 92 (2015), 104032, 21, arXiv: 1510.05593 [gr-qc]. MR 3465140

[4] H. Bondi, M.G.J. van der Burg, and A.W.K. Metzner, Gravitational waves in general relativity VII: Waves from axisymmetric isolated systems, Proc. Roy. Soc. London A 269 (1962), 21-52. MR MR0147276 (26 \#4793)

[5] P.T. Chruściel and L. Ifsits, The cosmological constant and the energy of gravitational radiation, Phys. Rev. D 93 (2016), 124075 (40 pp.), arXiv:1603.07018 [gr-qc].

[6] K. Fischer, Interpretation of Einstein's theory of gravitation including the cosmological term as a de Sitter-invariant field theory on the de Sitter space, Z. Physik 229 (1969), 33-43. MR 0255216
[7] H. Friedrich, On the existence of n-geodesically complete or future complete solutions of Einstein's field equations with smooth asymptotic structure, Commun. Math. Phys. 107 (1986), 587-609. MR 868737

[8] T. Mädler and J. Winicour, Bondi-Sachs Formalism, Scholarpedia 11 (2016), 33528, arXiv:1609.01731 [gr-qc].

[9] R.K. Sachs, Gravitational waves in general relativity VIII. Waves in asymptotically flat spacetime, Proc. Roy. Soc. London A 270 (1962), 103-126. MR MR0149908 (26 \#7393)

[10] V.L. Saw, Bondi mass with a cosmological constant, Phys. Rev. D 97 (2018), 084017, 14, arXiv:1711.01808 [gr-qc]. MR 3844732

[11] L.B. Szabados and P. Tod, A review of total energy-momenta in GR with a positive cosmological constant, Int. Jour. Mod. Phys. D 28 (2019), 1930003, arXiv:1808.09265 [gr-qc]. MR 3900124

[12] A. Trautman, Radiation and boundary conditions in the theory of gravitation, Bull. Acad. Pol. Sci., Série sci. math., astr. et phys. VI (1958), 407-412, arXiv:1604.03145. 